\title{
CONTINUA IN WHICH ALL CONNECTED SUBSETS ARE ARCWISE CONNECTED
}

BY

\author{
E. D. TYMCHATYN $\left({ }^{(}\right)$
}

\begin{abstract}
Let $X$ be a metric continuum such that every connected subset of $X$ is arcwise connected. Some facts concerning the distribution of local cutpoints of $X$ are obtained. These results are used to prove that $X$ is a regular curve.
\end{abstract}

1. Introduction. Several attempts have been made to characterize the spaces in which all connected subsets are arcwise connected, e.g. Kuratowski and Knaster [1], Whyburn [3], [5], [6] and Tymchatyn [2]. In [10] Mohler conjectured that if $X$ is a metric continuum such that each connected subset of $X$ is arcwise connected, then $X$ is a regular curve. The main purpose of this paper is to resolve this conjecture in the affirmative.

The reader may consult Whyburn $[8, \mathrm{~V} .2]$ for a survey of the properties of hereditarily locally connected continua and [8, III. 9] for a treatment of local cutpoints. Our notation follows Whyburn [8]. We collect here some basic definitions for the convenience of the reader. A continuum is a nondegenerate, compact, connected metric space. A continuum is said to be hereditarily locally connected if each of its subcontinua is locally connected. A continuum is said to be regular if it has a basis of open sets with finite boundaries. An arc is a homeomorph of the closed unit interval $[0,1]$. If $A$ is an arc and $c, d \in A$ then $[c, d]$ denotes the $\operatorname{arc}$ in $A$ with endpoints $c$ and $d$. A subset $A$ of a space $X$ is said to be arcwise connected if every pair of points of $A$ can be joined by an arc in $A$. A point $p$ in a continuum $X$ is said to be a local cutpoint or local separating point of $X$ if there is a connected open set $U$ in $X$ such that $U-\{p\}$ is not connected. The term neighbourhood will always mean open neighbourhood. We denote the closure of a set $A$ by $\mathrm{Cl}(A)$.

I wish to thank Professor A. Lelek and Professor L. Mohler for several helpful communications regarding this problem.

Received by the editors July 24, 1973 and, in revised form, February 14, 1974.

AMS (MOS) subject classifications (1970). Primary 54F50; Secondary 54F20, 54F25.

Key words and phrases. Regular curves, arcwise connected, continua, hereditarily locally connected.

(1) This research was supported in part by a grant from the National Research Council of Canada. 
2. In this section, we study several conditions that are satisfied by continua whose connected subsets are arcwise connected. The following theorem has played a central role in the study of these continua.

THEOREM 1 (Tymchatyn [2]). Let $X$ be a continuum such that $X=A_{0} \cup$ $A_{1} \cup \cdots$ where

(i) $A_{0}$ is not empty and contains no local cutpoints of $X$,

(ii) for each $i=1,2, \cdots, A_{i}$ is a closed set,

(iii) for each $i \neq j, A_{i} \cap A_{j}$ is void.

Then $X$ contains a connected set that is not arcwise connected.

LEMMA 2. If $X$ is a locally connected continuum such that $L$, the set of local cutpoints of $X$, is totally disconnected then $L$ can be written as the union of a countable family of pairwise disjoint closed sets.

Proof. It is well known (see $\left[8\right.$, p. 63]) that $L$ is an $F_{\sigma}$. Since $L$ is totally disconnected it is easy to see that $L$ can be written as the union of a countable family of pairwise disjoint closed sets.

By a null sequence of sets is meant a sequence of sets whose diameters converge to zero.

THEOREM 3. Let $X$ be a continuum such that every sequence of disjoint subcontinua of $X$ is a null sequence. Then the following are equivalent:

(a) If $A_{1}, A_{2}, \cdots$ is any sequence of pairwise disjoint closed subsets of $X$ and $x$ and $y$ are points that are separated by $X-\left(A_{1} \cup A_{2} \cup \cdots\right)$ then some countable subset of $X-\left(A_{1} \cup A_{2} \cup \cdots\right)$ separates $x$ and $y$ in $X$.

(b) If $A$ is any subcontinuum of $X$ and $x, y \in A$ then there is an arc in $A$ which contains $x$ and $y$ and which contains at most countably many points that are not local cutpoints of $A$.

(c) If $A$ is any subcontinuum of $X$ then the set of local cutpoints of $A$ is not contained in the union of countably many pairwise disjoint closed proper subsets of $A$.

Proof. (a) $\Rightarrow$ (c). Suppose (c) fails. Then there is a subcontinuum $A$ of $X$ such that the set of local cutpoints of $A$ is contained in the union of a countable family $A_{1}, A_{2}, \cdots$ of closed, proper, pairwise disjoint subsets of $A$. We may suppose without loss of generality that $A_{1}$ and $A_{2}$ are nonempty sets. Let $x \in A_{1}$ and let $y \in A_{2}$. Since every sequence of pairwise disjoint subcontinua of $X$ is a null sequence we may suppose that the $A_{i}$ form a null sequence. It follows that the decomposition space $Y$ obtained from $A$ by identifying each of the sets $A_{i}$ to a point is a compact metric space. The image of the set $A_{1} \cup A_{2} \cup \cdots$ 
in $Y$ under the natural projection $\pi$ is a countable set. Hence $A-\left(A_{1} \cup A_{2} \cup \cdots\right)$ separates $x$ and $y$ in $A$ since $Y-\pi\left(A_{1} \cup A_{2} \cup \cdots\right)$ separates $\pi(x)$ from $\pi(y)$ in $Y$. By [8,III. 9.41] every set in $A-\left(A_{1} \cup A_{2} \cup \cdots\right)$ which separates $x$ and $y$ is uncountable. In particular every set in $X$ which separates $x$ and $y$ is uncountable. Thus, (a) also fails.

(b) $\Rightarrow$ (c). Suppose (c) fails. Then there is a subcontinuum $A$ of $X$ such that the set of local cutpoints of $A$ is contained in the union of a countable family $A_{1}, A_{2} \cdots$ of closed proper subsets of $A$. By Sierpinski's theorem there exist $x$, $y \in A-\left(A_{1} \cup A_{2} \cup \cdots\right)$ and each arc in $A$ which contains $x$ and $y$ contains uncountably many points of $A-\left(A_{1} \cup A_{2} \cup \cdots\right)$. Hence, every arc in $A$ which contains $x$ and $y$ contains uncountably many points that are not local cutpoints of $A$. Thus, (b) also fails.

(c) $\Rightarrow$ (b). Suppose (b) fails. Let $L$ denote the set of local cutpoints of $X$. We may suppose without loss of generality that there exist $x, y \in X$ such that each arc in $X$ which contains both $x$ and $y$ contains uncountably many points of $X-L$. For each $z \in X$ let $A(z)=\bigcup\{A \subset X \mid A$ is a continuum, $z \in A$ and $A-L$ is countable\}.

By [7, Theorem 34] each $A(z)$ is closed. Clearly, $A(z)$ is also connected. Define an equivalence relation $\sim$ on $X$ by setting $x \sim y$ if and only if $x \in A(y)$. Since the set of nondegenerate equivalence classes of $\sim$ is a null sequence, it follows that $\sim$ is a closed relation.

Let $\pi$ be the natural projection of $X$ onto the quotient space $X / \sim . X$ is hereditarily locally connected since it can contain no continuum of convergence. Hence, $X / \sim$ is also a Peano continuum. A point $z \in X / \sim$ is a local cutpoint of $X / \sim$ only if $\pi^{-1}(z)$ contains a local cutpoint of $X$. Also, if $p$ is a local cutpoint of $X$ and $A(p)=\{p\}$ then $\pi(p)$ is a local cutpoint of $X / \sim$. We shall prove that the set of local cutpoints of $X / \sim$ is totally disconnected. By Lemma 2 it will follow that the set of local cutpoints of $X / \sim$ is the union of a countable family of pairwise disjoint closed sets and hence the set of local cutpoints of $X$ is contained in the union of a countable family of pairwise disjoint closed sets. Thus, it will have proved that (c) also fails.

Just suppose that the set of local cutpoints of $X / \sim$ is not totally disconnected. Since $X / \sim$ is a Peano continuum the set of local cutpoints of $X / \sim$ is an $F_{\sigma}$. It follows by the Sum Theorem for dimension zero that the set of local cutpoints of $X / \sim$ contains a continuum $A$. Then $\pi^{-1}(A)$ is a continuum in $X$ which is the union of uncountably many equivalence classes of $\sim$. Let $c, d \in \pi^{-1}(A)$ such that $c \nsucc d$. Let $C$ be an arc in $\pi^{-1}(A)$ with endpoints $c$ and $d$. For convenience, we identify $C$ with the closed unit interval $[0,1]$ with its usual order and its usual metric. To prove the theorem, it will suffice to prove that there is 
an $\operatorname{arc} D \subset \pi^{-1}(A)$ such that $c, d \in D$ and $D-L$ is at most countable.

Let $A_{1}, A_{2}, \cdots$ denote the nondegenerate equivalence classes of $\sim$. If $x \in C-L$ then $x \in A_{1} \cup A_{2} \cup \cdots$. Let $m_{1}$ be an integer such that diameter $\left(A_{m_{1}} \cap C\right) \geqslant$ diameter $\left(A_{i} \cap C\right)$ for each $i=1,2, \cdots$. Let $c_{i}=\min \left(A_{m_{1}} \cap C\right)$ and let $d_{1}=\max \left(A_{m_{1}} \cap C\right)$. Let $B_{1}$ be an arc in $A_{m_{1}}$ with endpoints $c_{1}$ and $d_{1}$ such that $B_{1}-L$ is at most countable. Notice that $A_{m_{1}} \cap C \subset\left[c_{1}, d_{1}\right]$ and for each $i=1,2, \cdots$ there do not exist $a, b \in A_{i} \cap C$ such that $a<c_{1}<d_{1}<b$.

Suppose $A_{m_{1}}, \cdots, A_{m_{n}}$ have been selected and for each $j=1, \cdots, n$, $B_{j}$ is an arc in $A_{m j}$ with endpoints $c_{j}$ and $d_{j}$ such that $B_{j}-L$ is at most countable and $\left(A_{m_{j}} \cap C\right) \subset\left[c_{1}, d_{1}\right] \cup \cdots \cup\left[c_{j}, d_{j}\right]$. Suppose also that there do not exist $k \in\{1, \cdots, n\}$ and an integer $i \in\{1,2, \cdots\}$ with $a, b \in\left(C \cap A_{i}\right)-$ $\left(\left[c_{1}, d_{1}\right] \cup \cdots \cup\left[c_{n}, d_{n}\right]\right)$ such that $a<c_{k}<d_{k}<b$. Suppose the intervals $\left[c_{i}, d_{i}\right]$ are disjoint.

If, for each $j,\left(A_{j} \cap C\right)-\left(\left[c_{1}, d_{1}\right] \cup \cdots \cup\left[c_{n}, d_{n}\right]\right)$ contains at most one point, then it is easy to check that $D=\left(C-\left(\left[c_{1}, d_{1}\right] \cup \cdots \cup\left[c_{n}, d_{n}\right]\right)\right)$ $\cup B_{1} \cup \cdots \cup B_{n}$ is an arc in $\pi^{-1}(A)$ which contains $c$ and $d$ and $D-L$ is at most countable.

Let us suppose, therefore, that there exists an integer $m_{n+1}$ such that

$$
\begin{aligned}
0 & <\operatorname{diameter}\left(\left(A_{m_{n+1}} \cap C\right)-\left(\left[c_{1}, d_{1}\right] \cup \cdots \cup\left[c_{n}, d_{n}\right]\right)\right) \\
& \geqslant \operatorname{diameter}\left(\left(A_{j} \cap C\right)-\left(\left[c_{1}, d_{1}\right] \cup \cdots \cup\left[c_{n}, d_{n}\right]\right)\right)
\end{aligned}
$$

for each $j=1,2, \cdots$.

Let

$$
c_{n+1}=\min \left(\left(A_{m_{n+1}} \cap C\right)-\left(\left[c_{1}, d_{1}\right] \cup \cdots \cup\left[c_{n}, d_{n}\right]\right)\right)
$$

and let

$$
d_{n+1}=\max \left(\left(A_{m_{n+1}} \cap C\right)-\left(\left[c_{1}, d_{1}\right] \cup \cdots \cup\left[c_{n}, d_{n}\right]\right)\right) .
$$

Let $B_{n+1}$ be an arc in $A_{m_{n+1}}$ with endpoints $c_{n+1}$ and $d_{n+1}$ such that $B-L$ is at most countable.

Let $D=\left(C-\left(\left[c_{1}, d_{1}\right] \cup\left[c_{2}, d_{2}\right] \cup \cdots\right)\right) \cup B_{1} \cup B_{2} \cup \cdots$. For each positive integer $i, A_{i} \cap\left(D-\left(\left[c_{1}, d_{1}\right] \cup\left[c_{2}, d_{2}\right] \cup \cdots\right)\right)$ contains at most one point. Thus, $D-L$ is at most countable.

For each positive integer $j$ let $h_{j}:\left[c_{j}, d_{j}\right] \rightarrow B_{j}$ be a homeomorphism such that $h_{j}\left(c_{j}\right)=c_{j}$ and $h_{j}\left(d_{j}\right)=d_{j}$. Define $h: C \rightarrow D$ by

$$
h(x)= \begin{cases}x & \text { if } x \notin\left[c_{j}, d_{j}\right] \text { for any } j, \\ h_{j}(x) & \text { if } x \in\left[c_{j}, d_{j}\right]\end{cases}
$$


Then $h$ is easily seen to be a homeomorphism of the $\operatorname{arc} C$ onto $D$. Thus $D$ is an arc in $\pi^{-1}(A)$ which contains $c$ and $d$ and $D-L$ is at most countable. Thus, $\pi(c)=\pi(d)$ which is a contradiction.

(c) $\Rightarrow$ (a). Suppose (a) fails. Then there is a sequence $A_{1}, A_{2}, \cdots$ of pairwise disjoint proper closed subsets of $X$ and points $x$ and $y$ that are separated by $X-\left(A_{1} \cup A_{2} \cup \cdots\right)$ such that no countable subset of $X-\left(A_{1} \cup A_{2} \cup \cdots\right)$ separates $x$ and $y$ in $X$. Let $A=A_{1} \cup A_{2} \cup \cdots$. We shall prove that $X$ contains a subcontinuum $B$ such that $x, y \in B$, no countable subset of $B-A$ separates $x$ and $y$ in $B$ and $B-A$ contains only countably many local cutpoints of $B$. Clearly the set of local cutpoints of $B$ is contained in the union of countably many disjoint proper closed sets so (c) fails.

We define by transfinite induction a nest of subcontinua $B_{\alpha}$ ) of $X$ as follows: Let $B_{0}=X$. Let $\alpha$ be a countable ordinal number. Suppose that for each $n<\alpha$, $B_{n}$ has been defined to be a subcontinuum of $X$ such that $x, y \in B$ and no countable subset of $B_{n}-A$ separates $x$ and $y$ in $B_{n}$. If, for some $n<\alpha, B_{n}-A$ contains only countably many local cutpoints of $B_{n}$, we are done. Suppose, therefore, that for each $n<\alpha, B_{n}-A$ has uncountably many local cutpoints of $B_{n}$.

Case 1. $\alpha$ is the successor of an ordinal number $m$. By assumption $B_{m}-A$ contains uncountably many local cutpoints of $B_{m}$. It follows from [8, III. 9.21] that every uncountable set of local cutpoints of a continuum contains a pair of points that separate the continuum. Thus, there exist $a_{\alpha}, b_{\alpha} \in B_{m}-(A \cup\{x, y\})$ such that $B_{m}-\left\{a_{\alpha}, b_{\alpha}\right\}$ is not connected. Let $B_{\alpha}$ be the closure of the component of $B_{m}-\left\{a_{\alpha}, b_{\alpha}\right\}$ that contains $x$ and $y$. Then, no countable set of $B_{\alpha}-A$ separates $x$ and $y$ in $B_{\alpha}$.

Case 2. $\alpha$ is a limit ordinal. Let $B_{\alpha}=\bigcap_{n<\alpha} B_{n}$. We shall show that no countable subset of $B_{\alpha}-(A \cup\{x, y\})$ separates $x$ and $y$ in $B_{\alpha}$. Let $C$ be a countable subset of $B_{\alpha}$.

Let $C^{\prime}=C \cup \bigcup_{n<\alpha}\left\{a_{n+1}, b_{n+1}\right\}$. Since $C^{\prime}$ is countable $x$ and $y$ lie in the same component $E$ of $X-C^{\prime}$. There is an $\operatorname{arc} D$ in the locally connected, topologically complete, metric space $E$ with endpoints $x$ and $y$. By induction it is easy to see that $D \subset B_{n}$ for each $n<\alpha$. Hence $D \subset B_{\alpha}-C$ which implies that $x$ and $y$ lie in the same component of $B_{\alpha}-C$.

Since $X$ does not contain uncountably many pairwise disjoint nondegenerate subcontinua it follows that for some countable ordinal $\alpha, B_{\alpha}$ has at most countably many local cutpoints of $B_{\alpha}$ in $B_{\alpha}-A$. This completes the proof of Theorem 3.

Corollary III. 9.21 in Whyburn [8] asserts that if $X$ is a metric continuum and if $G$ is an uncountable set of local cutpoints of $X$ then there is a countable subset $G_{0}$ of $G$ such that every point of $G-G_{0}$ is of order 2 relative to $G-G_{0}$. In particular, there exist $a, b \in G-G_{0}$ such that $X-\{a, b\}$ is not connected. 
It is easy to see that $X-\{a, b\}$ has either 2 or 3 components.

If $X$ is as above and $C$ is a connected subset of $X$ then $\mathrm{Cl}(C)-C$ contains at most countably many local cutpoints of $\mathrm{Cl}(C)$. For let $G$ be an uncountable set of local cutpoints of $\mathrm{Cl}(C)$. Let $a, b \in G$ such that $\mathrm{Cl}(C)-\{a, b\}$ is not connected. Since $C$ is connected and dense in $\mathrm{Cl}(C)$ it follows that $C-\{a, b\}$ is not connected. Thus, at least one of $a$ and $b$ is in $C$ and $G \not \subset \mathrm{Cl}(C)-C$.

In order that all of the connected subsets of a regular continuum should be arcwise connected it is necessary that the continuum satisfy conditions (a)-(b) of Theorem 3. If $X$ is a regular continuum that satisfies condition (a) of Theorem 3 , let $Y$ be a connected set in $X$. Let $D$ be a countable dense set in $Y$ and let $a \in D$. By Theorem 3 for each $d \in D$ there is an $\operatorname{arc} A_{d}$ in $\mathrm{Cl}(Y)$ such that $a, d \in A_{d}$ and $A_{d}$ contains at most countably many points that are not local cutpoints of $\mathrm{Cl}(Y)$. By the last paragraph $A_{d}-Y$ is at most countable. By [7, Theorem 34] $Y \cup \cup\left\{A_{d} \mid d \in A\right\}$ is arcwise connected. Thus, there is a countable set $C=\bigcup\left\{A_{d}-Y \mid d \in A\right\}$ such that $Y \cup C$ is arcwise connected.

Question 1. If $X$ is a regular continuum that satisfies condition (a) of Theorem 3, is every connected subset $Y$ of $X$ arcwise connected?

Question 2. If $X$ is as in Question 1, is every connected $F_{\sigma}$ in $X$ arcwise connected? In particular is the set of local cutpoints of $X$ arcwise connected if it is connected?

Question 3. Let $X$ be a regular continuum such that every pair of separated sets in $X$ can be separated by a countable set. Is every connected subset of $X$ arcwise connected?

THEOREM 4. Let $X$ be a regular continuum. If $C$ is a connected subset of $X$ then $C$ cannot be decomposed into countably infinitely many pairwise disjoint sets that are closed in $C$.

Proof. Let $C$ be a connected subset of $X$. Suppose $C=\bigcup_{i=1}^{\infty} A_{i}$ where the $A_{i}$ are pairwise disjoint proper subsets of $C$ which are closed in $C$. Since every sequence of pairwise disjoint connected sets in a regular space is a null sequence we may suppose the sequence $A_{i}$ ) is a null sequence.

Define an equivalence relation $\sim$ on $C$ by letting $x \sim y$ if and only if there is a natural number $i$ such that $x, y \in A_{i}$. Let $\pi$ be the natural projection of $C$ onto $C / \sim$. Now $C / \sim$ with the quotient topology is a connected countable space. We shall obtain a contradiction by proving that $C / \sim$ cannot be connected because it is a countable, $T_{1}$, normal space. It is clear that $C / \sim$ is a $T_{1}$ space since $\pi^{-1}(p)$ is closed in $C$ for each $p \in C / \sim$. It remains to prove only that $C / \sim$ is normal.

Let $M$ and $N$ be disjoint closed sets in $C / \sim$. Then $\pi^{-1}(M)$ and $\pi^{-1}(N)$ 
are disjoint closed sets in $C$. Since $C$ is normal there exist disjoint open sets $U$ and $V$ in $C$ such that $\pi^{-1}(M) \subset U$ and $\pi^{-1}(N) \subset V$. Since the sequence $\left.A_{i}\right)$ is a null sequence of closed sets in $X$ it follows that $\pi^{-1}(\pi(C-U))$ is a closed set in $C$ which misses $\pi^{-1}(M)$. Hence, $U-\pi^{-1}(\pi(C-U))$ and $V-\pi^{-1} \pi(C-V)$ are neighbourhoods of $\pi^{-1}(M)$ and $\pi^{-1}(N)$ respectively whose images under $\pi$ are disjoint neighbourhoods of $M$ and $N$ respectively in $C / \sim$.

COROLlary 5. A connected subset of a regular continuum has either one or uncountably many arc components.

Proof. The arc components of a connected subset of a regular continuum are closed (see [7, p. 334]); hence Theorem 4 applies.

3. Continua that are not regular. For the remainder of this section let $X$ be a fixed continuum that is not regular but which contains no nonnull sequence of pairwise disjoint subcontinua.

Definition. Let $M$ be a subcontinuum of a continuum $X$ and let $x, y \in M$. We say $M$ satisfies property $P(x, y)$ if no finite set separates $x$ and $y$ in any neighbourhood of $M$.

THEOREM 6. If $X$ is a continuum that is not regular then $X$ contains a connected subset that is not arcwise connected.

Proof. We may suppose as in [2] that $X$ is a hereditarily locally connected continuum and that every sequence of pairwise disjoint subcontinua of $X$ is a null sequence. By Lemma 2 we may suppose that for each subcontinuum $P$ of $X$ the set of local cutpoints of $P$ is not totally disconnected.

Let $a \in X$ such that $X$ is not regular at $a$. Let $M$ denote the set of points of $X$ which cannot be separated from $a$ by a finite set in $X$. By Whyburn [8, V. 4.4, 4.5] $M$ is a nondegenerate continuum. By Whyburn [8, III. 9.2] at most a countable number of points of $M$ are local cutpoints of $X$.

The first three claims can be proved by contradiction. The proofs are straightforward and are omitted.

Claim 1. $M$ satisfies $P(a, b)$ for each $b \in M-\{a\}$.

Let $b \in M-\{a\}$. Let $\left.M_{\lambda}\right)_{\lambda \in \Lambda}$ be a maximal nest of subcontinua of $M$ each of which satisfies property $P(a, b)$ and let $P_{a b}=\bigcap_{\lambda \in \Lambda} M_{\lambda}$. Then $P_{a b}$ is a continuum which is irreducible with respect to satisfying $P(a, b)$, for if $U$ is a neighbourhood of $P_{a b}$ then $M_{\lambda} \subset U$ for some $\lambda \in \Lambda$ and hence no finite set separates $a$ and $b$ in $U$. We shall describe in great detail the structure of the continuum $\boldsymbol{P}_{a b}$. 
In [2, Theorem 6] the author considered the case where $P_{a b}$ is an arc. To see that $P_{a b}$ may be quite complicated let $Y$ be the continuum constructed in the proof of Theorem 6 in [2]. Then $Y=A_{0} \cup A_{1} \cup A_{2} \cup \cdots$ where $A_{1}, A, \cdots$ are pairwise disjoint arcs and $A_{0}$ is also an arc. Let $Z$ be the decomposition space obtained from $Y$ by contracting $A_{i}$ to a point for each $i=1,2, \cdots$. Then $Z=$ $P_{a b}$ where $a$ and $b$ are the endpoints of the arc $A_{0}$.

Claim 2. If $c, d \in P_{a b}$ then $P_{a b}$ satisfies $P(c, d)$.

Claim 3. If $U$ is a neighbourhood of $x \in P_{a b}$ such that $b \notin U$ then there is a point $c$ in the boundary of $U$ and a continuum $P \subset P_{a b}-U$ such that $P$ satisfies $P(b, c)$.

If $x \in P_{a b}$ is not a local cutpoint of $X$ and $Q$ and $P$ are continua in $P_{a b}$ which satisfy $P(a, x)$ and $P(x, b)$ respectively then $P \cup Q$ is a continuum satisfying $P(a, b)$ and so $P \cup Q=P_{a b}$ by the irreducibility of $P_{a b}$.

CLAIM 4. If $x \in P_{a b}-\{b\}$ then there exists a proper subcontinuum of $P_{a b}$ which satisfies $P(a, x)$.

Proof. Suppose the claim is false. Let $x \in P_{a b}-\{b\}$ such that no proper subcontinuum of $P_{a b}$ satisfies $P(a, x)$.

If $U$ is any neighbourhood of $b$ in $P_{a b}$ then $\mathrm{Cl}(U)-U$ contains at most countably many local cutpoints of $\mathrm{Cl}(U)$ by the argument following Theorem 3. If $U$ is connected, then by Lemma 2 there exists an $\operatorname{arc} I$ of local cutpoints of $\mathrm{Cl}(U)$. Now $I$ intersects the boundary of $U$ in a set that is compact and at most countable so $U$ contains an arc of local cutpoints of $U$ and hence of $P_{a b}$.

By the last paragraph, there exists a sequence $I_{i}$ ) of pairwise disjoint arcs in $P_{a b}-\{b\}$ such that $\lim \sup I_{i}=\{b\}$ and for each $i=1,2, \cdots$ each point of $I_{i}$ is a local cutpoint of $P_{a b}$.

Let $c_{i}$ and $d_{i}$ be the endpoints of $I_{i}$. Since $P_{a b}$ contains only countably many local cutpoints of $X$ we may suppose $c_{i}$ and $d_{i}$ are not local cutpoints of $X$. By the argument following Theorem 3 we may suppose that $c_{i}$ and $d_{i}$ are points of order 2 in $P_{a b}$, that $c_{i}$ and $d_{i}$ separate $P_{a b}$ into either 2 or 3 components and that the component $K_{i}$ of $P_{a b}-\left\{c_{i}, d_{i}\right\}$ which meets $I_{i}$ contains neither $a$ nor $b$. By Whyburn $[3, \S 4]$ we may suppose that every point of $I_{i}-\left\{c_{i}, d_{i}\right\}$ disconnects $K_{i}$. Let (by [8, III. 9.21]) $z_{i} \in I_{i}-\left\{c_{i}, d_{i}\right\}$ be a point of order 2 in $P_{a b}$ such that $z_{i}$ is not a local cutpoint of $X$. Then $z_{i}$ separates $K_{i}$ into exactly two components. Finally, we may assume that the sets $K_{i}$ ) are pairwise disjoint. For each $i$ let $U_{i}$ be a neighbourhood of $K_{i}$ such that $\mathrm{Cl}\left(U_{i}\right) \cap \mathrm{Cl}\left(U_{j}\right)$ is empty for $i \neq j$. Notice that $\mathrm{Cl}\left(K_{i}\right)=K_{i} \cup\left\{c_{i}, d_{i}\right\}$. 
By Claim 3 there is a component $P$ of $P_{a b}-K_{i}$ such that $P$ satisfies either $P\left(a, c_{i}\right)$ or $P\left(a, d_{i}\right)$. We may suppose without loss of generality that $P$ satisfies $P\left(a, c_{i}\right)$. Since $c_{i}$ is not a local cutpoint of $X$ it follows that $P$ does not satisfy $P\left(c_{i}, b\right)$ for otherwise $P$ would be a proper subcontinuum of $P_{a b}$ which satisfies $P(a, b)$. By Claim 3 it follows that there is a component $Q$ of $P_{a b}-K_{i}$ such that $Q$ satisfies $P\left(d_{i}, b\right)$. Let $P_{a c_{i}} \subset P$ and $P_{d_{i} b} \subset Q$ be continua which are irreducible with respect to satisfying $P\left(a, c_{i}\right)$ and $P\left(d_{i}, b\right)$ respectively. If $P_{a c_{i}} \cap P_{d_{i} b}$ is infinite let $U$ be any neighbourhood of $P_{a c_{i}} \cup P_{a d_{i}}$. Let $A$ be a finite set in $U$ and let $x \in\left(P_{a c_{i}} \cap P_{d_{i} b}\right)-A$. By Claim 2, $P_{a c_{i}}$ satisfies $P(a, x)$ and $P_{a d_{i}}$ satisfies $P(x, b)$. Since $U$ is a neighbourhood of both $P_{a c_{i}}$ and $P_{a d_{i}}, A$ does not separate $a$ from $x$ or $x$ from $b$ in $U$. Thus $A$ does not separate $a$ from $b$ in $U$ and so $P_{a c_{i}} \cup \boldsymbol{P}_{d_{i} b}$ is a proper subcontinuum of $P_{a b}$ which satisfies $P(a, b)$. With this contradiction we conclude that $P_{a c_{i}} \cap P_{d_{i} b}$ is finite. A similar argument can be used to show that every point of $P_{a c_{i}} \cap P_{d_{i} b}$ is a local cutpoint of $X$.

By using the facts that $P_{a b}-K_{i}$ does not satisfy $P(a, b)$ while $P_{a b}$ satisfies $P(a, b)$, that $\mathrm{Cl}\left(K_{i}\right)-K_{i}=\left\{c_{i}, d_{i}\right\}$ and that $c_{i}$ and $d_{i}$ are not local cutpoints of $X$ one can easily prove by contradiction that $\mathrm{Cl}\left(K_{i}\right)$ satisfies $P\left(c_{i}, d_{i}\right)$. Since $c_{i}$ and $d_{i}$ are not local cutpoints of $X$ it follows from the remark following Claim 3 that $P_{a b}=P_{a c_{i}} \cup K_{i} \cup P_{d_{i} b}$.

Since $z_{i}$ separates $c_{i}$ and $d_{i}$ in $\mathrm{Cl}\left(K_{i}\right)$ it follows from Claim 3 that $\mathrm{Cl}\left(K_{i}\right)$ satisfies $P\left(c_{i}, z_{i}\right)$. Since $c_{i}$ is not a local cutpoint of $X, P_{a c_{i}} \cup \mathrm{Cl}\left(K_{i}\right)$ satisfies $P\left(a, z_{i}\right)$. Let $P_{a z_{i}} \subset P_{a c_{i}} \cup \mathrm{Cl}\left(K_{i}\right)$ be a continuum which is irreducible with respect to satisfying $P\left(a, z_{i}\right)$.

Since $b \notin P_{a c_{i}} \cup \mathrm{Cl}\left(K_{i}\right)$ we may suppose that, for each $i, z_{i+1} \in P_{d_{i} b}$. Hence, $\mathrm{Cl}\left(K_{i+1}\right) \subset P_{d_{i} b}$. By Claim 2 we may suppose that $P_{d_{i+1} b} \subset P_{d_{i} b}-K_{i+1}$. It now follows that $P_{a z_{i}} \subset P_{a c_{i}} \cup \mathrm{Cl}\left(K_{i}\right) \subset P_{a c_{i+1}} \subset P_{a z_{i+1}}$.

Since $P_{a b}$ is irreducible with respect to satisfying $P(a, x)$ it follows that $x \notin P_{a z_{i}}$ for each $i$ but $x \in \lim \sup P_{a z_{i}}=P_{a b}$. We may suppose that for each $i$ there is $x_{i} \in P_{a z_{i}}-P_{a z_{i-1}}$ such that $\lim x_{i}=x$. Since $P_{a b}$ contains at most countably many local cutpoints of $X$ we may suppose each $x_{i}$ is not a local cutpoint of $X$.

For each $i=2,3, \cdots$ there is a continuum $P_{d_{i-1} c_{i+1}} \subset P_{d_{i-1} b} \cap P_{a c_{i+1}}$ such that $P_{d_{i-1} c_{i+1}}$ is irreducible with respect to satisfying $P\left(d_{i-1}, c_{i+1}\right)$. Notice that $x_{i}, z_{i} \in P_{d_{i-1} c_{i+1}}$.

For each $i$ let $W_{i}$ be a neighbourhood of $P_{a b}-K_{i}$ and $A_{i}$ a finite set such that $A_{i}$ separates $a$ and $b$ in $W_{i}$ and $W_{i+j} \subset W_{i} \cup U_{i}$ for all $i, j=1,2, \cdots$. Since $x_{i}$ and $z_{i}$ are not local cutpoints of $X$ we may suppose that $x_{i}, z_{i} \notin A_{j}$ for all $i$ and $j$. Note that for $j=1,2, \cdots, z_{i+j}$ and $x_{i+j}$ lie in the component of $W_{i}-A_{i}$ 
which contains $b$ and, for $j=1, \cdots, n-1, z_{i-j}$ and $x_{i-j}$ lie in the component of $W_{i}-A_{i}$ which contains $a$.

For each $i=2,4,6, \cdots, P_{d_{i-1} c_{i+1}} \subset W_{1} \cap \cdots \cap W_{i} \cap W_{i+1}$ so there exists an arc $C_{i}$ joining $x_{i}$ to $z_{i}$ in $\left(W_{1} \cap \cdots \cap W_{i-1} \cap W_{i+1}\right)-\left(A_{1} \cup \cdots \cup\right.$ $\left.A_{i-1} \cup A_{i+1}\right)$. The arcs $C_{i}$ are pairwise disjoint by construction. This contradicts our assumption that every sequence of disjoint subcontinua of $X$ is a null sequence. The claim is proved.

Claim 5. If $x \in P_{a b}$ then there is a unique continuum $P_{a x}$ (resp. $\left.P_{x b}\right)$ in $P_{a b}$ which is irreducible with respect to satisfying $P(a, x)($ resp. $P(x, b))$.

Proof. Just suppose $A$ and $B$ are two subsets of $P_{a b}$ which are irreducible with respect to satisfying $P(a, x)$. Let $y \in A-B$ and let $z \in B-A$ such that $y$ and $z$ are not local cutpoints of $X$. Let $P_{y b} \subset P_{a b}$ be a continuum which is irreducible with respect to satisfying $P(y, b)$. By the remark following Claim 3 $P_{a b}=A \cup P_{y b}$ thus $z \in P_{y b}$. By Claim 4 there exists a continuum $\boldsymbol{P}_{z b} \subset \boldsymbol{P}_{y b}-$ $\{y\}$ such that $P_{z b}$ satisfies $P(z, b)$. Thus, $B \cup P_{z b}$ is a proper subcontinuum of $P_{a b}$ which satisfies $P(a, b)$. This is a contradiction. The claim is proved.

Claim 6. If $x, y \in P_{a b}$ then either $P_{a x} \subset P_{a y}$ or $P_{a y} \subset P_{a x}$.

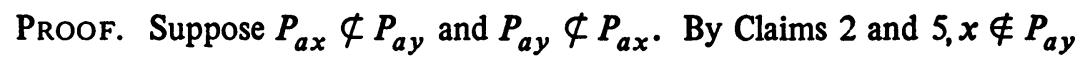
and $y \notin P_{a x}$. Since $P_{a b}$ contains at most countably many local cutpoints of $X$, we may suppose $x$ and $y$ are not local cutpoints of $X$.

Since $P_{a x} \cup P_{x b}=P_{a b}, y \in P_{x b}$. By Claim 4, $P_{y b} \nsubseteq P_{x b}$. Thus, $x \notin P_{y b}$ and $P_{a y} \cup P_{y b}$ is a proper subcontinuum of $P_{a b}$ which satisfies $P(a, b)$. This is a contradiction. The claim is proved.

Let $2^{P_{a b}}$ denote the space of closed subsets of $P_{a b}$ with the Hausdorff metric topology. Let $C$ denote the closure in $2^{P_{a b}}$ of $\left\{P_{a x} \mid x \in P_{a b}\right\}$.

CLAIM 7. $C$ is homeomorphic to the closed unit interval $[0,1]$.

Proof. $2^{P_{a b}}$ is a compact metric space that is partially ordered by inclusion. This partial order is a closed relation on $2^{P_{a b}}$. Since $C$ is compact, we need only prove that $C$ is connected and totally ordered under inclusion (Ward [9]).

Let $C \in C$. We start by showing that if $x \in C$ then $P_{a x} \subset C$. For let $x_{i}$ ) be a sequence in $P_{a b}$ such that $P_{a x_{i}}$ ) converges to $C$ in $C$. If, for arbitrarily large $i, x \in P_{a x_{i}}$ then $P_{a x} \subset P_{a x_{i}}$ for all such $i$ by Claims 2 and 5 and hence $\boldsymbol{P}_{a x} \subset \lim \sup \boldsymbol{P}_{a x_{i}}=C$. If, on the other hand, $\boldsymbol{P}_{a x_{i}} \subset \boldsymbol{P}_{a x}$ for arbitrarily large $i$ and if $U$ is a neighbourhood of $C$ such that $a$ and $x$ can be separated by a finite 
set in $U$, then for some sufficiently large $i P_{a x_{i}}$ can be separated in $U$ by that same finite set. This contradicts Claim 2 . By Claim 6 , these are the only two cases we need consider. Thus, $x \in C$ implies $P_{a x} \subset C$.

Let $C, D \in C$ such that $C \not \subset D$. Let $x \in C-D$ such that $x$ is not a local cutpoint of $X$ and let $y \in D$. Then $x \notin P_{a y}$ since $P_{a y} \subset D$. By Claim $6 y \in$ $P_{a x} \subset D$. Thus $D \subset C$ and $C$ is totally ordered by inclusion.

If $C$ is not connected then there exist $C, D \in C$ with $C \subsetneq D$ such that for each $E \in C$ either $E \subset C$ or $D \subset E$. Now, $D-C$ contains at least two points $x$ and $y$ such that $x$ and $y$ are not local cutpoints of $X$. Then $P_{a x}=P_{a y}=D$. By Claim $4 x=y$. With this contradiction we conclude that $C$ is connected. The claim is proved.

Claim 8. If $x_{i}$ ) is a sequence in $P_{a b}$ such that the sequence $P_{a x_{i}}$ ) converges in $\left(\right.$ then $x_{i}$ ) converges in $P_{a b}$.

Proof. Just suppose that $x_{i}$ ) and $y_{i}$ ) are two sequences in $P_{a b}$ which converge to $x$ and $y$ respectively such that the sequences $\left.P_{a x_{i}}\right)$ and $\left.P_{a y_{i}}\right)$ both converge to $C \in C$. We may suppose that, for each $i, x_{i}$ ) and $y_{i}$ ) are not local cutpoints of $X$ and neither sequence is constant.

By Claim 6 we need to consider only three cases.

Case 1. For each $i, P_{a x_{i}} \subset P_{a x_{i+1}}$ and $P_{a y_{i}} \subset P_{a y_{i+1}}$. If, for each $i$ and $j, P_{a x_{i}} \subset P_{a y_{j}}$ then $C=P_{a y_{1}}$ and the sequence $y_{i}$ ) would have to be constant. We may suppose, therefore, that for each $i P_{a x_{i}} \subset P_{a y_{i}} \subset P_{a x_{i+1}}$. As in the proof of Claim 4 if $y \in P_{a x_{i}} \cap P_{x_{i} b}$ and $y$ is not a local cutpoint of $X$ then $y=x_{i}$. Since $P_{a b}$ contains at most countably many local cutpoints of $X, P_{a x_{i}} \cap$ $\boldsymbol{P}_{x_{i} b}$ is at most a countable set. Since $\boldsymbol{P}_{a y_{i-1}} \nsubseteq \boldsymbol{P}_{a x_{i}}$ it follows that $\boldsymbol{P}_{a b}-$ $\left(P_{x_{i} b} \cup P_{a y_{i-1}}\right)$ is a nonempty open subset of $P_{a b}$ which is contained in $P_{a x_{i}}$. As in Claim 4 there exists an arc $I_{i}$ of local cutpoints of $P_{a b}$ such that $I_{i} \subset P_{a b}$ $\left(P_{x_{i} b} \cup P_{a y_{i-1}}\right) \subset P_{a x_{i}}-P_{a y_{i-1}}$. Now construct as in Claim 4 a sequence $\left.A_{i}\right)$ of pairwise disjoint arcs such that for each $i x_{i}, y_{i} \in A_{i}$. Since every sequence of disjoint continua in $X$ is null, $x=y$.

Case 2. For each $i P_{a x_{i}} \supset P_{a x_{i+1}}$ and $P_{a y_{i}} \supset P_{a y_{i+1}}$.

Argue as in Case 1.

Case 3. For each $i P_{a x_{i}} \subset P_{a x_{i+1}}$ and $P_{a y_{i}} \supset P_{a y_{i+1}}$. Then $P_{a x_{i}} \subset P_{a y_{j}}$ for each $i$ and $j$. Let $U$ be a neighbourhood of $x$ in $P_{a b}$ such that the boundary of $U$ contains no local cutpoints of $X$ and $y \notin U$. For each $i$ let $z_{i} \in P_{x_{i} y_{i}} \cap$ (boundary of $U$ ). Then, $P_{a x_{i}} \subset P_{a z_{i}} \subset P_{a y_{i}}$ so that $\lim P_{a z_{i}}=C$.

If for some subsequence $z_{i_{j}}$ ) $P_{a z_{i j}} \subsetneq P_{a z_{i j+1}}$ for each $j$, then we are in Case 1 with the sequences $x_{i}$ ) and $z_{i_{j}}$ ). If for some subsequence $\left.z_{i_{j}}\right) P_{a z_{i_{j}}} \supsetneq P_{a z_{i_{j+1}}}$ 
for each $j$ then we are in Case 2 with the sequences $z_{i_{j}}$ ) and $y_{i}$ ). If the sequence $z_{i}$ ) contains a constant subsequence we may suppose $z_{i}=z$ for each $i$. Let $w_{i}$ ) be a sequence in $P_{a z}$ which converges to $z$. Then $\lim P_{a w_{i}}$ $=P_{a z}=C$. We may now apply Case 1 to the sequences $w_{i}$ ) and $x_{i}$ ).

The claim is now proved.

We are now in a position to adapt the proof of Theorem 6 in [2] to the continuum $P_{a b}$.

We shall attach to $P_{a b}$ an infinite sequence of pairwise disjoint closed sets $A_{i}$ such that no pair of points of $P_{a b}$ can be separated by a finite set in $P_{a b} \cup$ $\left(\cup A_{i}\right)$.

Let $h$ be a homeomorphism of the closed unit interval $[0,1]$ onto $C$ such that $h(0)=\{a\}$ and $h(1)=P_{a b}$. Define $f:[0,1] \rightarrow P_{a b}$ by letting

$$
f(r)= \begin{cases}x, & \text { if } h(r)=P_{a x}, \\ \lim x_{i}, & \text { if } h(r)=\lim P_{a x_{i}}\end{cases}
$$

By Claim $8 f$ is a continuous function. Notice that if $x \in P_{a b}$ is not a local cutpoint of $X$ then $f^{-1}(x)$ is a singleton.

If $A$ is a set in $X$ and $\epsilon>0$ we let $S(A, \epsilon)$ denote the $\epsilon$-neighbourhood of $A$ in $X$.

By the proof of Claim 4 if $r \in[0,1]$, then $h(r)=\{f(r)\} \cup(U\{h(s) \mid s<r\})$.

Let $0<r<1$. For $\epsilon>0$ there exists, by the proof of Claim 4, $\delta>0$ such that $r<s<\delta+r$ implies $h(s) \subset h(r) \cup S(f(r), \epsilon)$.

Let $Y_{r}=\bigcup\left\{P_{x b} \mid x \in P_{a b}-h(r)\right\}$. Let $\left.r_{i}\right)$ be a sequence in $[0,1]$ which is strictly decreasing to $r$ such that, for each $i, f\left(r_{i}\right)$ is not a local cutpoint of $X$. It can be shown as in the proof of Claim 4 that $Y_{r}=\bigcup P_{f\left(r_{i}\right) b}$ and $Y_{r} \cup\{f(r)\}$ is compact.

If $0<s<1$ and $U$ is any neighbourhood of $h(s)$ then no pair of points of $h(s)$ can be separated in $U$ by a finite set. We may suppose therefore that either

(1) for each $i$ there exist $c_{i}, d_{i} \in[0,1]$ with $c_{i}<r<d_{i}$ such
that $f\left(c_{i}\right)=f\left(d_{i}\right) \in\left(h(r) \cap Y_{r} \cap S(f(r), 1 / i)\right)-\{f(r)\}$, or

for each $i$ there exists an arc $C_{i} \subset S(f(r), 1 / i)$ such that $C_{i} \cap h(1)$ consists precisely of the two endpoints of $C_{i}$,

(2) one endpoint of $C_{i}$ is in $h(r)-\{f(r)\}$ and the other is in $h(1)-h(r)$. The arcs $C_{i}$ may be taken to be pairwise disjoint.

Suppose (1) holds. Let $E_{i}=\left\{f\left(c_{i}\right)\right\}$. We wish to show that $\lim c_{i}=r$. Just suppose that for each $i c_{i} \leqslant s<r$. Then $h(s) \cup Y_{r}$ is a continuum in $P_{a b}$ 
which satisfies $P(a, b)$. If $s<t<r$ and $f(t)$ is not a local cutpoint of $X$ then $f(t) \notin h(s) \cup Y_{r}$. This contradicts the assumption that $P_{a b}$ is an irreducible continuum with respect to satisfying $P(a, b)$. Thus, $\lim c_{i}=r$. Similarly, $\lim d_{i}=r$.

Now suppose (2) holds. Since every sequence of disjoint subcontinua of $X$ is a null sequence, there exists a sequence $\epsilon_{i}$ ) of positive numbers converging to zero such that if $D_{i}$ is the component of $\left[S\left(h(1), \epsilon_{i}\right) \cup C_{i}\right]-h(1)$ which meets $C_{i}$ then $D_{i} \cap D_{j}=\varnothing$ for $i \neq j$ and the diameters of the $D_{i}$ converge to 0 .

Let $M_{i}=\mathrm{Cl}\left(D_{i}\right) \cap(h(r)-\{f(r)\})$ and $N_{i}=\mathrm{Cl}\left(D_{i}\right) \cap\left(Y_{r}-\{f(r)\}\right)$. If $M_{i}$ (resp. $N_{i}$ ) has an isolated point let $c_{i}$ (resp. $d_{i}$ ) be an isolated point of $M_{i}$ (resp. $\left.N_{i}\right)$. If $M_{i}$ (resp. $\left.N_{i}\right)$ has no isolated points let $c_{i}=\inf \left\{s \in[0,1] \mid \mathrm{Cl}\left(D_{i}\right)\right.$ $\cap h(s)$ is uncountable $\}$ (resp. $d_{i}=\sup \left\{s \in[0,1] \mid \mathrm{Cl}\left(D_{i}\right) \cap P_{f(s) b}\right.$ is uncountable $\}$ ). Then $h\left(c_{i}\right) \cap \mathrm{Cl}\left(D_{i}\right)$ is at most countable.

As in (1) $\lim c_{i}=\lim d_{i}=r$. Let $E_{i}$ be an $\operatorname{arc}$ in $D_{i} \cup\left\{c_{i}, d_{i}\right\}$ with endpoints $c_{i}$ and $d_{i}$.

We wish to prove that for each $\epsilon>0$ and each $s \in[0,1]$ either

$$
\left(h(s) \cap Y_{s} \cap S(f(s), \epsilon)\right)-\{f(s)\} \neq \varnothing
$$

or there exists an arc in $S(f(s), \epsilon)-E_{i}$ which joins $h(s)$ to $h(1)-h(s)$. We need only consider $s \in[0,1]$ such that $f(s)=f\left(c_{i}\right)$ or $f(s)=f\left(d_{i}\right)$.

Clearly, (**) is satisfied if both $M_{i}$ and $N_{i}$ have an isolated point. Suppose, therefore, that $M_{i}$ does not have an isolated point. Let $s \in[0,1]$ such that $f(s)=f\left(c_{i}\right)$ and suppose $\epsilon>0$ is given such that

$$
\left(h(s) \cap Y_{s} \cap S(f(s), \epsilon)\right)-\{f(s)\}=\varnothing .
$$

By (*) there is a sequence of $\operatorname{arcs} F_{j} \subset S(f(s), \epsilon)-\{f(s)\}$ which join $h(s)$ to $h(1)-h(s)$ such that $\lim F_{j}=\{f(s)\}$. Let $e_{j}$ and $f_{j}$ be the endpoints of $F_{j}$. We may suppose $F_{j} \cap h(1)=\left\{e_{j}, f_{j}\right\}$ where $e_{j} \in h(s)$ and $f_{j} \in h(1)-h(s)$.

Just suppose that for each $j E_{i} \cap F_{j} \neq \varnothing$. Then each $e_{j} \in \mathrm{Cl}\left(D_{i}\right)$. By the choice of $c_{i}$ and by the assumption that $M_{i}$ is a perfect set each neighbourhood of $e_{j}$ contains uncountably many points of $Y_{c_{i}}$. Since $Y_{c_{i}} \cup\left\{f\left(c_{i}\right)\right\}$ is compact $e_{j} \in Y_{c_{i}}$. If $s \leqslant c_{i}$ then

$$
e_{j} \in\left(h(s) \cap Y_{s} \cap S(f(s), \epsilon)\right)-\{f(s)\}
$$

which is a contradiction. If $c_{i}<s$ then for each $j$ let $e_{j}^{\prime} \in[0,1]$ such that $f\left(e_{j}^{\prime}\right)=e_{j}$. We get as in (1) that $\lim e_{j}^{\prime}=s$ and hence eventually $e_{j} \in Y_{s}-h\left(c_{i}\right)$ which is again a contradiction. We conclude that for all sufficiently large $j$ $E_{i} \cap F_{j}=\varnothing$. Thus, (**) is satisfied.

If there exist $c<1 / 2<d$ such that $f(c)=f(d) \in S(f(1 / 2), 1)-\{f(1 / 2)\}$ let $C(1 / 2,1)=\{f(c)\}$. Otherwise, let $C(1 / 2,1)$ be an arc in $S(h(1), 1)$ with endpoints 
$f(c)$ and $f(d)$ where $1 / 4<c<1 / 2<d<3 / 4$ and $C(1 / 2,1)$ is obtained as was $E_{i}$ above.

Let $A_{1}=C(1 / 2,1)$. Suppose $A_{1}, \cdots, A_{n-1}$ have been constructed to be pairwise disjoint closed sets such that for each $i=1, \cdots, n-1$

(i) $A_{i}$ is the union of a finite number of arcs and points each of which was obtained as was $E_{i}$ above,

(ii) for each $a \in\left[1 / 2^{i}, 1-1 / 2^{i}\right]$ there is a component $C$ of $A_{i}$ such that $C$ meets both $f\left(\left[1 / 2^{i}, a\right]\right)-\{f(a)\}$ and $f\left(\left[a, 1-1 / 2^{i}\right]\right)-\{f(a)\}$,

(iii) $P_{a b} \cap A_{i} \supset P_{a b}-f\left(\left[0,1 / 2^{i+1}\right] \cup\left[1-1 / 2^{i+2}, 1\right]\right)$.

For each $x \in\left[1 / 2^{n}, 1-1 / 2^{n}\right]$ let $C(x, n) \subset S\left(h(1), 1 / 2^{n-1}\right)-\left(A_{1} \cup \cdots\right.$ $\cup A_{n-1}$ ) be an (possibly degenerate) arc chosen as was $E_{i}$ with endpoints $f(c(x, n))$ and $f(d(x, n))$ where $1 / 2^{n+1}<c(x, n)<x<d(x, n)<1-1 / 2^{n+1}, C(x, n)$ minus its endpoints lies in $X-h(1)$ and $C(x, n)$ satisfies (**). The set of open intervals ] $c(x, n), d(x, n)\left[\right.$ such that $x \in\left[1 / 2^{n}, 1-1 / 2^{n}\right]$ is an open cover for the compact set $\left[1 / 2^{n}, 1-1 / 2^{n}\right]$ hence there exists a minimal finite set $\left\{x_{1}, \cdots, x_{k}\right\} \subset$ $\left[1 / 2^{n}, 1-1 / 2^{n}\right]$ such that

$$
] c\left(x_{1}, n\right), d\left(x_{1}, n\right)[\cup \cdots \cup] c\left(x_{k}, n\right), d\left(x_{k}, n\right)[
$$

covers $\left[1 / 2^{n}, 1-1 / 2^{n}\right]$. It is clear that conditions (i)-(iii) are satisfied for $n$. Let $A_{n}=C\left(x_{1}, n\right) \cup \cdots \cup C\left(x_{k}, n\right)$.

Let $Y=P_{a b} \cup A_{1} \cup A_{2} \cup \cdots$. Since every sequence of disjoint subcontinua of $X$ is a null sequence $Y$ is a continuum.

To prove that $Y$ contains a connected set that is not arcwise connected it suffices by Theorem 1 and the fact that $P_{a b}$ contains only countably many local cutpoints of $X$ to prove that if $z$ is a local cutpoint of $Y$ then either $z \in A_{i}$ for some $i$ or $z$ is a local cutpoint of $X$. Let $z \in P_{a b}$ such that $z$ is not a local cutpoint of $X$ and $z \notin A_{i}$ for any $i$. It follows from the fact that $f^{-1}(z)$ is a singleton that if $U$ is any connected neighbourhood of $z$ in $P_{a b}$ such that $U-\{z\}$ has more than one component then $U-\{z\}$ has exactly two components one of which is contained in $\left\{f(y) \mid y<f^{-1}(z)\right\}$ and the other is in $\left\{f(y) \mid f^{-1}(z)<y\right\}$. It is now easy to see that $z$ is not a local cutpoint of $Y$ and the theorem is proved.

\section{REFERENCES}

1. K. Kuratowski and B. Knaster, A connected and connected im kleinen point set which contains no perfect subset, Bull. Amer. Math. Soc. 33 (1927), 106.

2. E. D. Tymchatyn, Continua whose connected subsets are arcwise connected, Colloq. Math. 24 (1971/72), 169-174, 286. MR 46 \#9946.

3. G. T. Whyburn, On the existence of totally imperfect and punctiform connected sets in a given continuum, Amer. J. Math. 55 (1933), 146-152. 314.

4. _- Local separating points of continua, Monatsh. Math. Phys. 36 (1929), 305-

5. - Decompositions of continua by means of local separating points, Amer. J. Math. 55 (1933), 437-457. 
6. G. T. Whyburn, Sets of local separating points of a continuum, Bull. Amer. Math. Soc. 39 (1933), 97-100.

7. - Concerning points of continuous curves defined by certain im kleinen properties, Math. Ann. 102 (1930), 313-336.

8. - Analytic topology, Amer. Math. Soc. Colloq. Publ., vol. 28, Amer. Math. Soc., Providence, R. I., 1942. MR 4, 86.

9. L. E. Ward, Jr., Partially ordered topological spaces, Proc. Amer. Math. Soc. 5 (1954), 141-161. MR 16, 59.

10. L. Mohler, Cuts and weak cuts in metric continua, Proc. Conf. Point Set Topology (Univ. of Houston, Houston, Tex., 1971).

DEPARTMENT OF MATHEMATICS, UNIVERSITY OF SASKATCHEWAN, SASKATOON, SASKATCHEWAN S7N OWO CANADA 